\title{
Extremely Metal Poor Stars in the Galaxy
}

\author{
P. François \\ Paris-Meudon Observatory, 61 Avenue de l'Observatoire, F-75014 PARIS \\ email: patrick.francois@obspm.fr
}

\begin{abstract}
Globular Clusters and metal poor stars represent two aspects of the population II stars in our Galaxy. The presentation will focus on the metal poor end of population II of galactic field stars.
\end{abstract}

Keywords. stars : abundances, stars : population II, Galaxy : halo.

\section{Introduction}

It is well know that the metallicity distribution of galactic halo stars and globular clusters is very different and the link between the two is not trivial. Recent observations have shown that the single metallicity population paradigm for globular clusters is no more valid for some Globular Clusters for which very accurate photometry can be performed (Piotto et al., 2007). What can we say about galactic metal poor stars ?

\section{Metal Poor Stars and Extremely Metal Poor Stars}

The history of the study of metal poor stars is closely linked with the progress in observing facilities. As example, the early 80's abundance diagrams $[\mathrm{Ca} / \mathrm{Fe}]$ vs $[\mathrm{Fe} / \mathrm{H}]$ showed an over-solar value of this ratio in metal poor stars with some hints of an increasing value as metallicity decreases. A 10 years observational effort has permitted to confirm this progressive rise of the $[\mathrm{Ca} / \mathrm{Fe}]$ as $[\mathrm{Fe} / \mathrm{H}]$ decreases (Edvardsson et al. 1993). A more careful selection of metal poor stars thanks to kinematics has revealed the existence of substructures in the abundance diagrams $[\alpha /[\mathrm{Fe}]$ (Nissen \& Schuster 2010). These two distinct populations stars in the solar neighborhood belong to the metal-rich end of the halo metallicity distribution function. The standard single zone models of chemical evolution of the Galaxy where no or a small abundance dispersion is found at a given metallicity have to be extended to inhomogeneous models able to include these substructures. The recent discovery of extensive sub-structures (Belokurov et al. (2006) reveal that the halo has a more complex history than previously thought (Eggen et al. 1962, Searle et al., 1978). Carollo (2007) has recently shown that the halo can be divided of two broadly overlapping structural components-an inner and an outer halo with different spatial density profiles, stellar orbits and stellar metallicities. As extremely metal poor stars are rare, the extension of this work towards very low metallicities requires ambitious large scale spectroscopic surveys. Among them, we can mention the LAMOST project for which a dedicated telescope equipped with 16 spectrographs have been built with the aim of acquiring $10^{6}$ spectra. Another ongoing project is the Gaia-ESO survey (GES) which will use the FLAMES and UVES spectrographs with a goal of more than 2. $\times 10^{5}$ spectra for mostly $\mathrm{F}$ and G stars. Similar and complementary projects are foreseen in the near future (HERMES, APOGEE, 4MOST, MOONS to cite few of them).

These large spectroscopic surveys are also of paramount interest for their use to find the most metal poor stars in our Galaxy. These peculiar objects are identified from low to 
medium resolution spectra from large surveys (mainly H\&K BPS, Hamburg-ESO Survey \& Sloan Digital Sky Survey with SEGUE) by analyzing the strength of the Ca H\& K lines used a metallicity indicator and photometric or spectro-photometric indices used as a temperature estimator. In 2005, the most metal poor star known was HE 1327-2326 (Frebel et al. 2005). This star has a very high enhancement in Carbon. This enhancement has been also found in the top five most metal poor stars. Frebel et al. (2007) concluded that forming low-mass stars should have a minimum amount of Carbon and Oxygen in order to allow the cooling of the forming low mass star. The consequence was that all the most metal poor stars should be $\mathrm{C}$ and $\mathrm{O}$ enriched. Several programs to find these most metal poor stars are ongoing. They are based on a selection of candidates on the low $/ \mathrm{med}$ resolution spectroscopic surveys which are observed with high resolution spectroscopic observations (CASH, OZ, ...). This star formation cooling theory of low mass stars has been challenged by the discovery of a star Caffau et al. (2011) which has a metallicity $[\mathrm{Fe} / \mathrm{H}]=-5$ and no $\mathrm{C}$ enhancement falling in the range where stars could not form in the frame of Frebel's model of low-mass star formation.

\section{The ESO Large Programme: Turn Off PrimOrdial Stars (ToPoS)}

An ESO large program (ToPoS) led by E. Caffau has been initiated in 2012. This project has three main goals: to determine the metal-weak tail of the halo metallicity distribution function, below $[\mathrm{M} / \mathrm{H}]=-3.5$, where the low resolution SDSS spectra are inadequate, to determine the relative abundance of the elements in Extremely/Ultra metal poor stars, signature of the massive First stars and to determine the trend of the lithium abundance in the matter at the beginning of the Galaxy. Among the 9 stars observed so far, 4 have a $[\mathrm{Fe} / \mathrm{H}]$ below -3.5 dex in which a star with $[\mathrm{Fe} / \mathrm{H}]<-4.6$ dex. The results which will be published in a forthcoming paper demonstrate the efficiency of the target selection process.

\section{References}

Belokurov, V., Zucker, D. B., Evans, N. W., Gilmore, G., Vidrih, S., Bramich, D. M., Newberg, H. J., Wyse, R. F. G., Irwin, M. J., Fellhauer, M., Hewett, P. C., Walton, N. A., Wilkinson, M. I., Cole, N., Yanny, B., Rockosi, C. M., Beers, T. C., Bell, E. F., Brinkmann, J., Ivezić, Z̆., \& Lupton, R. 2006 ApJ 642, L137

Caffau, E., Bonifacio, P., François, P., Sbordone, L., Monaco, L., Spite, M., Spite, F., Ludwig, H.-G., Cayrel, R., Zaggia, S., Hammer, F., Randich, S., Molaro, P., \& Hill, V. 2006 Nature 477,67

Carollo, D., Beers, T. C., Lee, Y. S., Chiba, M., Norris, J. E., Wilhelm, R., Sivarani, T., Marsteller, B., Munn, J. A., Bailer-Jones, C. A. L., Fiorentin, P. R., \& York, D. G. 2007 Nature 450, 1020

Edvardsson, B., Andersen, J., Gustafsson, B., Lambert, D. L., Nissen, P. E., \& Tomkin, J. 1993, $A \& A 275,101$

Eggen, O. Lynden-Bell, D., \& Sandage, A. 1962, ApJ 137,748

Frebel, A., Aoki, W., Christlieb, N., Ando, H., Asplund, M., Barklem, P. S., Beers, T. C., Eriksson, K., Fechner, C., Fujimoto, M. Y., Honda, S., Kajino, T., Minezaki, T., Nomoto, K., Norris, J. E., Ryan, S. G., Takada-Hidai, M., Tsangarides, S., \& Yoshii, Y. 2005, Nature 434,871

Frebel, A., Johnson, J. L., \& Bromm, V. MNRAS 380, 40

Nissen, P. E., \& Schuster, W. J. (2010 ) 2010, A\&A 511, L10

Piotto, G., Bedin, L. R., Anderson, J., King, I. R., Cassisi, S., Milone, A. P., Villanova, S., Pietrinferni, A., \& Renzini, A. 2007, ApJ 661, L53

Searle, L. \& Zinn, R., 1978 1978, ApJ 225,357 